\title{
Hypoxic-ischemic injury results in acute disruption of myelin gene expression and death of oligodendroglial precursors in neonatal mice
}

\author{
Robert P. Skoff ${ }^{a, *}$, Denise A. Bessert ${ }^{a}$, John D.E. Barks ${ }^{b}$, Dekun Song ${ }^{b}$, \\ Mirela Cerghet $^{a}$, Faye S. Silverstein ${ }^{b, c}$ \\ a Department of Anatomy and Cell Biology, Wayne State University School of Medicine, 540 East Canfield, Detroit, MI 48201, USA \\ ${ }^{\mathrm{b}}$ Departments of Pediatrics, University of Michigan, Ann Arbor, MI 48109, USA \\ ${ }^{\mathrm{c}}$ Neurology, University of Michigan, Ann Arbor, MI 48109, USA
}

\begin{abstract}
Studies of ischemic brain injury in neonatal rodents have focused upon the pathophysiology of neuronal damage. Much less consideration has been given to white matter injury, even though it is a major contributor to chronic neurological dysfunction in children. In the human neonate, particularly in those born prematurely, periventricular white matter is highly susceptible to hypoxic-ischemic $(\mathrm{H}-\mathrm{I})$ injury. To understand the basis for this selective vulnerability, we examined myelin gene expression and cell death in the subventricular layer and the surrounding white matter of neonatal mice following $\mathrm{H}-\mathrm{I}$ insult. Using an in situ hybridization technique that gives high resolution and is very sensitive, we examined myelin basic protein and proteolipid protein gene expression three and twenty-four hours after a $\mathrm{H}-\mathrm{I}$ insult. To elicit unilateral forebrain hypoxic and ischemic injury, 9-10-day-old mice underwent right carotid artery ligation followed by timed (40-70 $\mathrm{min}$ ) exposure to 10\% oxygen. Twenty-four hours following $\mathrm{H}-\mathrm{I}$, myelin basic protein and proteolipid protein transcripts were markedly reduced in striatum, external capsule, fornix, and corpus callosum in the injured side. Three hours after lesioning (ligation +70 min hypoxic exposure) myelin basic protein gene transcripts were visibly reduced in the ipsilateral white matter tracts. Interestingly, some cells in the subventricular layer expressed proteolipid protein transcripts, and $3 \mathrm{~h}$ after a $\mathrm{H}-\mathrm{I}$ insult they were degenerating in the injured but not contralateral side. TUNEL staining showed an increase in the number of positive cells in the injured subventricular layer and corpus callosum but the adjacent striatum did not show a corresponding change in the number of TUNEL labeled cells. Ultrastructural studies of the subventricular zone and corpus callosum $3 \mathrm{~h}$ after $\mathrm{H}-\mathrm{I}$ revealed that many subventricular cells, glial cells in the corpus callosum, and callosal axons in the injured side had already degenerated. However, the subventricular cells, glia and axons in the contralateral corpus callosum were spared. Many cells in the injured corpus callosum exhibited a apoptotic morphology; yet more mature oligodendrocytes in this region appeared normal. Our results show that a $\mathrm{H}-\mathrm{I}$ insult causes a surprisingly swift and dramatic degenerative response in the subventricular layer and adjacent white matter. Within $3 \mathrm{~h}$ after $\mathrm{H}-\mathrm{I}$, the programmed cell death cascade was initiated; internucleosomal DNA degradation took place in subventricular and glial cells; oligodendrocyte progenitors died and axonal degeneration in the ipsilateral corpus callosum was extensive. The swiftness of the subventricular and glial cell degeneration suggests the $\mathrm{H}-\mathrm{I}$ insult directly targets glia, as well as neurons, and raises the provocative question of whether glia exert damaging effects upon neurons and axons. Since the severity of the $\mathrm{H}-\mathrm{I}$ insult can be modulated by varying the duration of hypoxia, the model is ideal to study whether oligodendrocyte progenitors are more susceptible to death than mature oligodendrocytes, whether mature oligodendrocytes de-differentiate and then are induced to remyelinate surviving axons, and/or whether oligodendrocyte progenitors in the subventricular layer can be stimulated to proliferate, migrate, and remyelinate the surviving axons. (c) 2001 ISDN. Published by Elsevier Science Ltd. All rights reserved.
\end{abstract}

Keywords: Oligodendrocyte; Hypoxia-ischemia; Apoptosis; Neuroglia; Myelin basic protein; Proteolipid protein

Abbreviations: H-I, hypoxia-ischemia; MBP, myelin basic protein; OL, oligodendrocyte; PLP, proteolipid protein; VZ, ventricular zone; SVZ, subventricular zone.

* Corresponding author. Tel.: + 1-313-5771165; fax: + 1-313-5773377.

E-mail address: rskoff@med.wayne.edu (R.P. Skoff). 


\section{Introduction}

Neonatal hypoxic-ischemic $(\mathrm{H}-\mathrm{I})$ white matter injury is a major contributor to chronic neurological dysfunction (Volpe, 1999). In the neonatal period, periventricular white matter is highly susceptible to ischemic injury. Periventricular leukomalacia (PVL) is a distinctive pattern of perinatal brain injury, characterized by gliosis and cystic degeneration of the deep white matter surrounding the lateral ventricles. PVL is the best-defined neuroanatomic correlate of spastic diplegia (a common form of cerebral palsy). PVL is most frequent in premature infants; the cerebral vasculature of the premature infant makes the periventricular white matter a 'watershed' zone that is highly susceptible to hypoperfusion. Recent experimental studies suggest that intrinsic properties of immature oligodendrocytes (OLs) render them particularly vulnerable to cerebral ischemia (Fern and Moller, 2000).

Several recent reports highlight the vulnerability of OLs to diverse mechanisms of injury that are relevant in the context of acute hypoxia-ischemia. Immature OLs were found to be significantly more sensitive to oxidative stress (mediated by glutathione depletion) than were mature OLs (Back et al., 1998). When immature OLs in vitro were deprived of oxygen and glucose (to model ischemia), over $80 \%$ of immature OLs died within $25 \mathrm{~min}$, and cell death occurred after $\mathrm{Ca}^{2+}$ influx mediated by non-NMDA glutamate receptors (Fern and Moller, 2000). Surprisingly, mature oligodendroglia were more tolerant of the same insult.

Studies performed in the context of understanding the pathogenesis of immune-mediated demyelination have emphasized the susceptibility of OLs to pro-inflammatory cytokine-mediated damage (Hartung et al., 1992). The deleterious effects of cytokines on OLs are developmentaly modified; the cytotoxic effects of TNF$\alpha$ and interferon- $\gamma$ are the greatest in immature OLs (Andrews et al., 1998). This latter finding is of particular interest in a clinical context as there is substantial evidence that links maternal intrauterine infection and a pro-inflammatory response in the peri-partum period to an increased risk for development of neonatal white matter injury (Dammann and Leviton, 1997).

Mature OLs may also be more vulnerable to in vivo ischemic injury than was previously appreciated. In adult rats, $10 \mathrm{~min}$ of global ischemia using the four vessel occlusion model, an insult that produces CA1 hippocampal neuronal death but minimal neuronal damage in other regions, resulted in substantial OL damage (Petito et al., 1998). These findings may model the delayed white matter damage following an anoxic insult in humans. In an adult mouse stroke model (middle cerebral artery occlusion), Mandai et al. (1997) found that the OL-specific proteolipid protein (PLP) message declined at $12 \mathrm{~h}$ in the ischemic core and was almost absent at $24 \mathrm{~h}$. This acute reduction in a myelin message demonstrated ischemia-induced oligodendroglial injury.

Based upon the adult rodent models, several experimental systems have been developed to study ischemic brain injury in neonatal rodents over the past 20 years. One of the best-characterized and widely used models developed by Vannucci and colleagues (Rice et al., 1981) elicits unilateral forebrain ischemic injury. Postnatal day 7 (P7) rats undergo unilateral carotid ligation followed by $1.5-3 \mathrm{~h}$ exposure to $8 \%$ oxygen $\left(\mathrm{O}_{2}\right)$. The combination of ligation and hypoxia are required to elicit forebrain ischemia and infarction. Although the original histological description of this model emphasized that myelinogenic foci were vulnerable, most research until recently has focused on the pathophysiology of neuronal damage. We have adapted this model to neonatal mice (Liu et al., 1999) using P9-10 mice that undergo right carotid ligation followed by moderate hypoxia $\left(10 \% \mathrm{O}_{2}, 40-120 \mathrm{~min}\right)$, and recently found that ipsilateral white matter is highly susceptible to injury, based on evaluation of myelin gene expression $24-48 \mathrm{~h}$ after lesioning (Silverstein et al., 2000).

White matter injury has been relatively neglected experimentaly, partly because of the lack of reliable indices of acute white matter injury, partly because of the difficulties inherent in quantification of white matter damage, and also the general perception that white matter injury is secondary to neuronal injury. In order to study mechanisms of white matter injury, it is critical to identify early changes in OL function that contribute to OL injury. This information can then be correlated with early changes in neuronal functions. Recently, we reported preliminary evidence that in $\mathrm{P} 7$ rat brain, two myelin-specific mRNA's encoding PLP and myelin basic protein (MBP) provided sensitive indices of acute oligodendroglial injury $24-48 \mathrm{~h}$ after lesioning (Xu et al., 2000).

The neonatal $\mathrm{H}-\mathrm{I}$ model is a particularly attractive system to study the plasticity of OLs and their progenitors. Since the severity of the $\mathrm{H}-\mathrm{I}$ insult can be modulated by altering the duration of hypoxia exposure, the model can be used to test whether immature OLs are more susceptible to death than mature OLs; whether mature OLs can dedifferentiate and then become reactivated to remyelinate surviving axons; and the extent of OL progenitors to proliferate, migrate, and remyelinate axons under various $\mathrm{H}-\mathrm{I}$ conditions. The ventricular zone (VZ)/subventricular zone (SVZ) is a likely source for generating OLs after injury and in postnatal development; VZ/SVZ cells are still migrating away from the VZ/SVZ to form myelinating cells of the corpus callosum and adjacent white matter tracts. Morphological identification of individual OL progenitors in VZ/SVZ prosencephalon has been difficult to achieve 
but by using a sensitive in situ hybridization assay, we can detect MBP and PLP mRNA expressing cells in the VZ/SVZ zone as early as E14 (Bessert and Skoff, 1999). These cells are putative sources to become actively involved in remyelination, and may be relevant to the classic neuropathological abnormality status marmoratus in which aberrant remyelination in the basal ganglia occurs following severe $\mathrm{H}-\mathrm{I}$ injury in the neonatal period. Accordingly, we examined whether $\mathrm{H}-\mathrm{I}$ injury induces an acute OL phenotype in $\mathrm{VZ} / \mathrm{SVZ}$ cells and correlated this data with TUNEL labeling and light and electron microscopy. Following a mild $\mathrm{H}-\mathrm{I}$ insult, we found that SVZ cells and OL progenitors undergo extensive degeneration within $3 \mathrm{~h}$ of the insult. However, actively myelinating OLs in the corpus callosum retained a normal morphology at this point. This observation supports tissue culture studies that show OL progenitors are more susceptible than adult OLs to an $\mathrm{H}-\mathrm{I}$ insult.

\section{Experimental procedures}

\subsection{Animals}

These experiments were carried out in $\mathrm{P} 9-10$ mice, weighing 4-5 gms. Animals had a mixed background of $129 / \mathrm{Sv}$ and C57BL/6-derived genes; this strain was used because we were interested in establishing lesion parameters for additional experiments in which they would serve as controls for geneticaly modified animals, bred on the same background strain (Liu et al., 1999). The animals underwent right common carotid artery ligation under isoflurane anesthesia. After a $1 \mathrm{~h}$ recovery period, mice were placed in $10 \% \mathrm{O}_{2}$ (balance nitrogen) in glass chambers partialy immersed in a $38^{\circ} \mathrm{C}$ water bath (chamber air temperature $37^{\circ} \mathrm{C}$ ) for 40,55 , or $70 \mathrm{~min}$. After hypoxia exposure, mice were observed in a heated incubator with air temperature maintained at $37^{\circ} \mathrm{C}$ for about $30 \mathrm{~min}$. When they resumed normal activity, they were returned to the dams. Three or $24 \mathrm{~h}$ after their exposure to hypoxia, lesioned animals and littermate controls received a lethal overdose of chloral hydrate $(300 \mathrm{mg} / \mathrm{kg})$. Two sets of mice, each set containing one or three normals and three experimental mice, were exposed for $70 \mathrm{~min}$ of hypoxia and examined $24 \mathrm{~h}$ after $\mathrm{H}-\mathrm{I}$. Three sets of mice, each set containing one to two normals and two to three experimental mice, were exposed for $70 \mathrm{~min}$ of hypoxia and examined $3 \mathrm{~h}$ later. Another group of mice consisted of two normal mice, two mice exposed for $40 \mathrm{~min}$ of hypoxia, two mice exposed for $55 \mathrm{~min}$ of hypoxia, and three mice exposed for $70 \mathrm{~min}$ of hypoxia, were sacrificed $3 \mathrm{~h}$ after the insult. The mice were perfused with ice-cold phosphate-buffered saline (PBS) followed with $4 \%$ paraformaldehyde. These experimental protocols were approved by the University of Michigan Committee on Use and Care of Animals. All efforts were made to minimize animal suffering and the number of animals used.

Brains were stored in $4 \%$ paraformaldehyde at $4{ }^{\circ} \mathrm{C}$. Previously, we showed in this model that ipsilateral forebrain ischemia is induced rapidly when animals are exposed to $10 \% \mathrm{O}_{2}$ (Liu et al., 1999), and that the duration of hypoxia exposure determines the severity of tissue injury. Assessment of routine histopathology (cresyl-violet stained $20 \mu \mathrm{m}$ frozen sections), 5 days after lesioning, reveals that $40 \mathrm{~min}$ exposure to $10 \% \mathrm{O}_{2}$ elicits minimal overt tissue damage, $70 \mathrm{~min}$ exposure elicits consistent ipsilateral neuronal damage and tissue loss in cortex, striatum, and hippocampus, and $120 \mathrm{~min}$ exposure typicaly elicits severe hemispheric infarction.

\subsection{In situ hybridization and immunocytochemistry}

The ventral right side of the brain was incised with a razor blade to identify the injured side on sections. Transverse serial Vibratomed sections $(50 \mu \mathrm{m})$ were cut at the level of the striatum, rostral hippocampus, and caudal hippocampus. Adjacent sections were processed for MBP or PLP in situ hybridization using the identical laboratory method detailed in a recent publication from our laboratories (Bessert and Skoff, 1999). Full length MBP and PLP cDNAs (kindly provided by A.T. Campagnoni, UCLA) were labeled with digoxigenin using the random primer method (Bessert and Skoff, 1999). Our previous studies showed that only OLs or their progenitors were stained using the digoxigenin labeled PLP and MBP probes. Elimination of the digoxigenin labeled probes from the in situ hybridization procedure showed no specific staining.

For MBP immunocytochemistry, a monoclonal antibody SMI94 (Sternberger Monoclonals, Lutherville $\mathrm{MD)}$ that recognizes all 4 major MBP isoforms was applied to free floating sections diluted 1:1000. Freefloating $(50 \mu \mathrm{m})$ sections were pretreated with $0.5 \%$ Triton in PBS for $1 \mathrm{~h}$, rinsed, incubated with the primary antibody for $1 \mathrm{~h}$ containing $0.1 \%$ Triton in M.O.M. diluent (Vector Laboratories, Burlingame CA). Sections were rinsed, incubated with biotinylated antimouse IgG following the manufacturer's directions (Vector Laboratories), rinsed, and mounted in Aquamount. Sections in some wells were treated as above except that the primary antibody was eliminated. These sections showed no specific staining of OLs or other cell types.

\subsection{TUNEL staining and plastic embedding}

In one series of animals, serial sections from some brains were processed for TUNEL, semi-thin and ultrathin microscopy, MBP and PLP in situ hybridization, 
and MBP immunocytochemistry. TUNEL staining was performed with a kit (Roche Molecular Biochemicals, Mannheim, Germany) according to manufacturers directions. For semi-thin and ultra-thin microscopy, 50 $\mu \mathrm{m}$ sections of the brain were osmicated, cut into
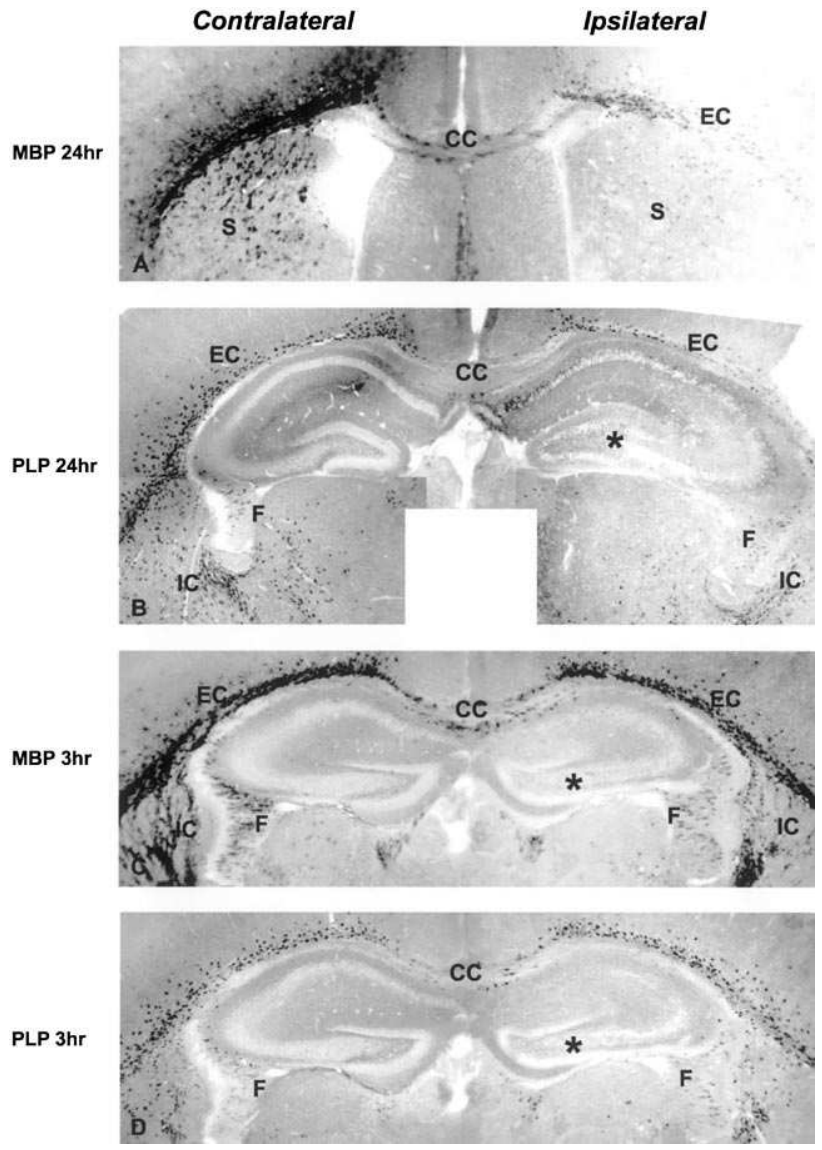

Fig. 1. Montages of 9-10-day-old mouse brains $24 \mathrm{~h}$ (A, B) or $3 \mathrm{~h} \mathrm{(C,}$ D) after lesioning. To elicit unilateral forebrain hypoxic-ischemic $(\mathrm{H}-\mathrm{I})$ injury, animals underwent right carotid artery ligation, followed by 70 min exposure to $10 \%$ oxygen. Vibratomed sections (50 $\mu \mathrm{m}$ ) were processed for MBP or PLP in situ hybridization (see Section 2). (A) At $24 \mathrm{~h}$ post $\mathrm{H}-\mathrm{I}$, MBP mRNA was dramaticaly decreased in striatum (S) and external capsule (EC) in the hemisphere ipsilateral to the carotid ligation. While the total message was decreased on the injured side, many cells in striatum and external capsule still expressed low levels of message. The corpus callosum (CC) bilateraly had few OLs expressing myelin messages. (B) At $24 \mathrm{~h}$ post $\mathrm{H}-\mathrm{I}$, PLP mRNA was decreased at level of hippocampus in the $\mathrm{H}-\mathrm{I}$ hemisphere. The most noticeable differences were in external capsule (EC), internal capsule (IC), and fornix (F). Tissue around Ammon's horn (asterisk) was degenerating. (C) At $3 \mathrm{~h}$ post $\mathrm{H}-\mathrm{I}$, MBP mRNA was moderately reduced in fornix (F) and internal capsule (IC), and the overall intensity of MBP mRNA in external capsule (EC) was moderately reduced in the ipsilateral versus the contralateral side. Corpus callosum (CC) contained small numbers of $\mathrm{MBP}^{+}$cells bilateraly. Organization of Ammon's horn (asterisk) was disrupted ipsilateraly. (D) At $3 \mathrm{~h}$ post $\mathrm{H}-\mathrm{I}$, differences in intensity of PLP transcripts between the ipsilateral and the contralateral side are not striking although intensity of message appears reduced ipsilateraly. Cellular organization of Ammon's horn (asterisk) was disrupted. This section was immediately adjacent to section above. $(\mathrm{A}-\mathrm{D}) \times 50$. eighths, and embedded in Araldite plastic using routine laboratory procedures. Semi-thin or ultra-thin sections were stained with Toluidine Blue/pyronin B or uranyl acetate/lead citrate, respectively. Electron micrographs were taken with a JEOL 1010.

\subsection{Microscopy}

Photomicrographs were taken with a Leitz Laborlux microscope using Kodak EliteChrome T160 slide film. To capture the whole cerebrum as many as 8 different pictures were taken using a $\times 2.5$ objective. These slides and electron micrographs were scanned with a Polaroid SprintScan 35 Plus and digitally imaged using Adobe Photoshop software. With the in situ hybridization and low magnification semi-thin sections, different areas of the same section were pasted together in Adobe Photoshop to generate montages of the brain. These color images were converted to black and white to increase the contrast.

\section{Results}

Twenty-four hours after ligation of right carotid artery and $70 \mathrm{~min}$ of hypoxia, both MBP and PLP transcripts were reduced in the right cerebral hemisphere. The most noticeable changes were found at the level of the striatum/septal nuclei and rostral hippocampus with attached fornix. The caudal hippocampus at the level of the thalamus was less severely affected. Myelin basic protein transcripts were markedly reduced in the external capsule, cingulum, and striatum in the injured side (Fig. 1A). The reduction of MBP transcripts was reflected in two ways; fewer OLs expressed MBP message, and intensity of reaction product was less in those OLs that still expressed MBP transcripts. Although the staining for MBP transcripts was dramaticaly reduced in the injured side, many $\mathrm{MBP}^{+}$cells in the striatum and external capsule continued to survive at this time point. Bilateraly in the corpus callosum, few cells expressed MBP and PLP transcripts $24 \mathrm{~h}$ post $\mathrm{H}-\mathrm{I}$. Unlesioned mice had more $\mathrm{MBP}^{+}$and $\mathrm{PLP}^{+}$cells in the corpus callosum at this age, and more myelin message bearing cells were found in the corpus callosum $3 \mathrm{~h}$ post $\mathrm{H}-\mathrm{I}$ (Fig. $1 \mathrm{C}-\mathrm{D})$. The reduction in the number of $\mathrm{MBP}^{+}$and $\mathrm{PLP}^{+}$cells ipsi- and contralateraly at $24 \mathrm{~h}$ after lesioning is possibly due to degeneration of OLs on both the sides or, alternatively, myelin messages in these OLs may be down regulated below the level of detection.

PLP transcripts were also down regulated in the external capsule, internal capsule and fornix in the right hemisphere $24 \mathrm{~h}$ post $\mathrm{H}-\mathrm{I}$ (Fig. 1B). The differences in intensity between MBP and PLP transcripts were primarily due to differences in localization of these tran- 


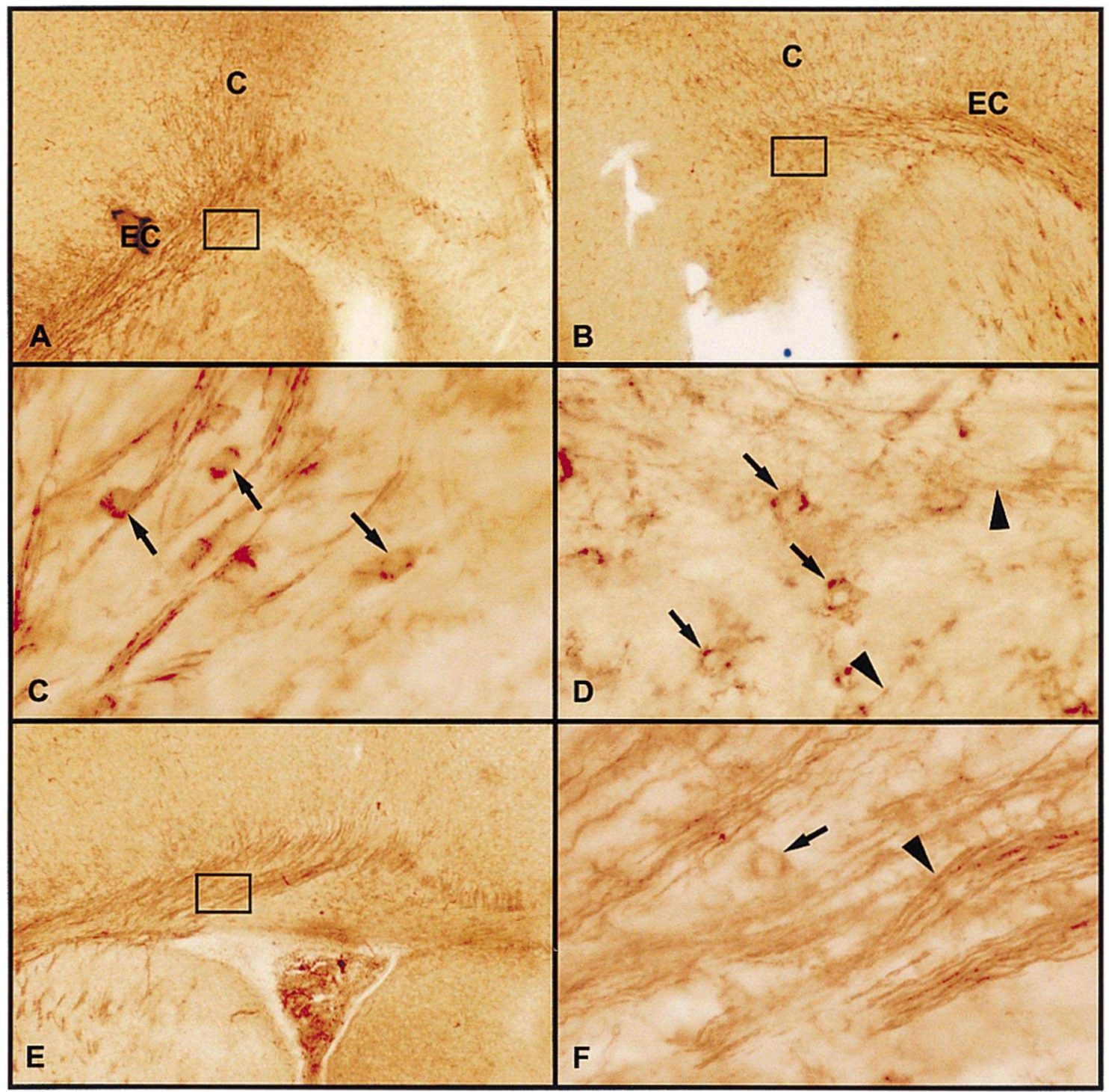

Fig. 2. MBP immunocytochemistry of 9-10-day-old mouse brains, evaluated $3 \mathrm{~h}$ after lesioning. To elicit unilateral forebrain $\mathrm{H}-\mathrm{I}$ injury, animals underwent right carotid artery ligation, followed by $70 \mathrm{~min}$ exposure to $10 \%$ oygen $(\mathrm{A}-\mathrm{D})$ or 40 min exposure (E-F). (A) The organization of fibers in the cingulum (C) and external capsule (EC) in the contralateral side exhibited the typical normal pattern. (C) Higher magnification of inset in Fig. 2A showed OL cell bodies (arrows) in close proximity to myelinated fibers. (B) At low magnification, the pattern of myelinated fibers in the ipsilateral side did not appear strikingly different from the contralateral side. (D) However, higher magnification of inset in Fig. 2B showed myelin is disrupted (arrowheads) and continuous internodes were difficult to identify. MBP immunostaining of OLs (arrows) retained a seemingly normal morphology. (E) In mildly lesioned animals (40 min $\mathrm{H}-\mathrm{I})$, the organization of the white matter tracts ipsilateral to ligation at level of striatum appeared normal. (F) Higher magnification showed myelinated fibers (arrowhead) with typical railroad track structure and several OLs (arrow). (A, B, E) $\times 30,(\mathrm{C}, \mathrm{D}, \mathrm{F}) \times 280$.

scripts. PLP transcripts were localized to the cell body whereas MBP transcripts extended into distal most OL processes and occupied much more area than PLP transcripts.

Three hours after right carotid ligation and $70 \mathrm{~min}$ of hypoxia, MBP transcripts were already reduced in the injured side (Fig. 1C) but not so noticeably as at $24 \mathrm{~h}$ post $\mathrm{H}-\mathrm{I}$. The reductions were most easily visualized in the internal capsule and fornix. The differences in the external capsule were less prominent but intensity of reaction product was already diminished. A serial sec- tion (Fig. 1D) adjacent to the section shown in Fig. 1C and processed for PLP in situ hybridization did not show pronounced changes between left and right sides.

A change in the level of MBP transcripts proved to be a more sensitive indicator than a change in the level of myelin basic protein at early time points post $\mathrm{H}-\mathrm{I}$. At low magnification, differences in MBP immunostaining between injured and uninjured sides were subtle and hardly noticeable $3 \mathrm{~h}$ after lesioning (carotid ligation +70 min $\left.10 \% \mathrm{O}_{2}\right)($ Fig. $2 \mathrm{~A}-\mathrm{B})$. At higher magnification, intact myelin sheaths were less frequently 
encountered but their remnants were still visible. Connections of OLs to myelin sheaths were difficult to find in the injured side, and the cell bodies were less intensely stained than controls. However, the shape of most OLs was normal (Fig. 2C and D). This observation is in agreement with ultrastructural studies that show many normal OLs are present in white matter tracts $3 \mathrm{~h}$ post $\mathrm{H}-\mathrm{I}$ (see below).

Plastic embedded sections $(1 \mu \mathrm{m})$ were prepared from mice sacrificed $3 \mathrm{~h}$ following right carotid ligation and $70 \min 10 \% \mathrm{O}_{2}$ exposure. At this level of resolution, it was apparent that the hippocampus and fornix were undergoing massive degeneration in the injured side (Fig. 3A). The region surrounding Ammon's horn was particularly affected (also Fig. 1B-D) but the cytoarchitecture of the whole hippocampus was disrupted. Higher magnificaton of the fornix in the injured side showed many axons have degenerated leaving fluid filled extracellular spaces. Surprisingly, many OLs with normal morphology were scattered throughout the fornix (data not shown); its cytoarchitecture $3 \mathrm{~h}$ post $\mathrm{H}-\mathrm{I}$ was similar to the corpus callosum (see below). Examination of semi-thin sections of brains from several mice that underwent $70 \mathrm{~min}$ of hypoxic-ischemic injury and that were sacrificed $3 \mathrm{~h}$ later showed similar degrees of hippocampal degeneration. These observations indicate the consistency of the $\mathrm{H}-\mathrm{I}$ protocol and resulted in similar degenerative changes from mouse to mouse.

The semi-thin sections revealed degenerative changes that were less easily visualized in the in situ hybridiza-

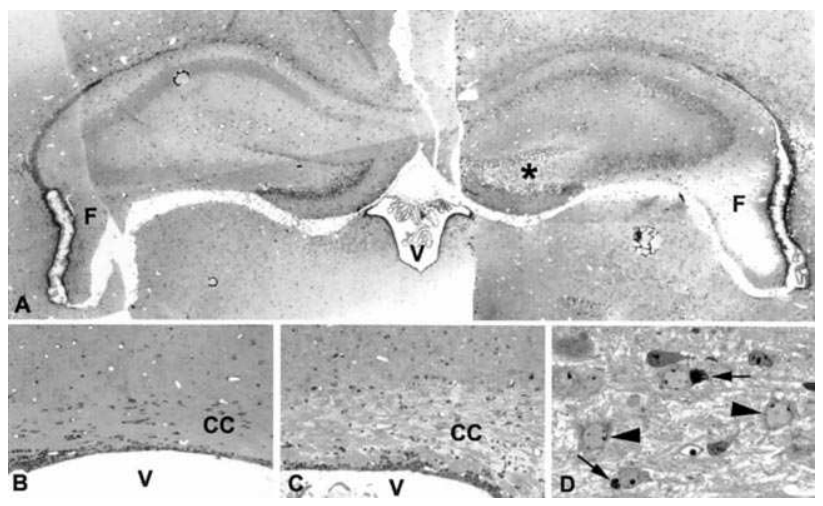

Fig. 3. Plastic embedded $(1 \mu \mathrm{m})$ sections of a P9 old mouse brain that was evaluated $3 \mathrm{~h}$ after lesioning. To elicit unilateral forebrain $\mathrm{H}-\mathrm{I}$ injury, the animal underwent right carotid artery ligation, followed by 70 min exposure to $10 \%$ oxygen. (A) Composite photographs of dorsal brain at level of third ventricle $(\mathrm{V})$. Degeneration of fornix $(\mathrm{F})$ was advanced on $\mathrm{H}-\mathrm{I}$ side (right) and cytoarchitecture of Ammon's horn is disrupted. (B-D) Sections more rostral to Fig. 3A from same animal showed corpus callosum (CC) adjacent to lateral ventricle (V) on contralateral (B) and lesioned (C) side. Degeneration of callosal fibers was already evident ipsilateraly; yet the layer of ventricular cells appeared normal on this side. (D) Higher magnification of the $\mathrm{H}-\mathrm{I}$ hemisphere showed degenerating cells (arrows) and several normal OLs (arrowheads). (A) $\times 30,(B, C) \times 70,(D) \times 360$. tion and immunocytochemical preparations. Right carotid artery ligation and $55 \mathrm{~min}$ of hypoxia followed by a $3 \mathrm{~h}$ survival showed extensive degenerative changes in the right Ammon's horn and fornix. Forty minutes of hypoxia followed by a 3-h survival elicited noticeable changes in the hippocampus of one of the two mice. However, MBP and PLP in situ hybridization and MBP immunocytochemistry of these mice did not show noticeable changes between uninjured and injured sides.

Three hours after lesioning, degeneration was limited principaly to the $\mathrm{H}-\mathrm{I}$ side of the brain. Semi-thin sections rostral to the section shown in Fig. 3A show the corpus callosum bilateraly. The corpus callosum and the ventricular layer in the uninjured side (Fig. 3B) had a normal morphology. In contrast, the corpus callosum on the opposite side (Fig. 3C) had fluid filled extracellular spaces. The ventricular layer itself appeared to be normal. Higher magnification of the injured side showed several degenerating cells but also, a surprising number of more mature, normal appearing OLs. Ultrastructural examination of the ipsi- and contralateral corpora callosa confirmed the light microscopic observations. In the uninjured corpus callosum, the organization of the ventricular/subventricular layers was normal (Fig. 4A). The integrity of the axons was intact and very few signs of degenerating axons were present (Fig. 4A and B). Deeper to the subventricular layer, the lamellar organization of myelinating axons was normal (Fig. 4B).

No degenerating cells were found in the ventricular layer in the lesioned hemisphere. However, the layer of subventricular cells immediately dorsal to the ventricle was missing. In the corpus callosum, axons no longer retained their organized longitudinal and transverse orientation. Many have presumably degenerated, and extracellular space was large (Fig. 4C-F). Many more degenerating cells were present in the injured corpus callosum than in the uninjured side. Some cells exhibited the classical features of apoptotic cells with condensed chromatin masses, and they had intact nuclear and plasma membranes and mitochondria with cristae (Fig. 4D). Other degenerating cells expressed most features of apoptotic cells but their cytoplasm appeared to be more swollen (Fig. 4E and F), and it is difficult based upon morphological criteria alone to state that they are apoptotic or necrotic. Although axonal degeneration in the corpus callosum was advanced $3 \mathrm{~h}$ post $\mathrm{H}-\mathrm{I}$, a surprising number of OLs had the morphology of actively myelinating cells, and showed only a modest increase in cytoplasmic inclusions.

The identity of the cells undergoing degeneration was difficult to determine at light and electron microscopic level. In the subventricular layer of control brains and in the hemisphere contralateral to ligation, lightly to moderately stained PLP message containing cells were present (Fig. 5A and B). PLP transcripts were restricted 


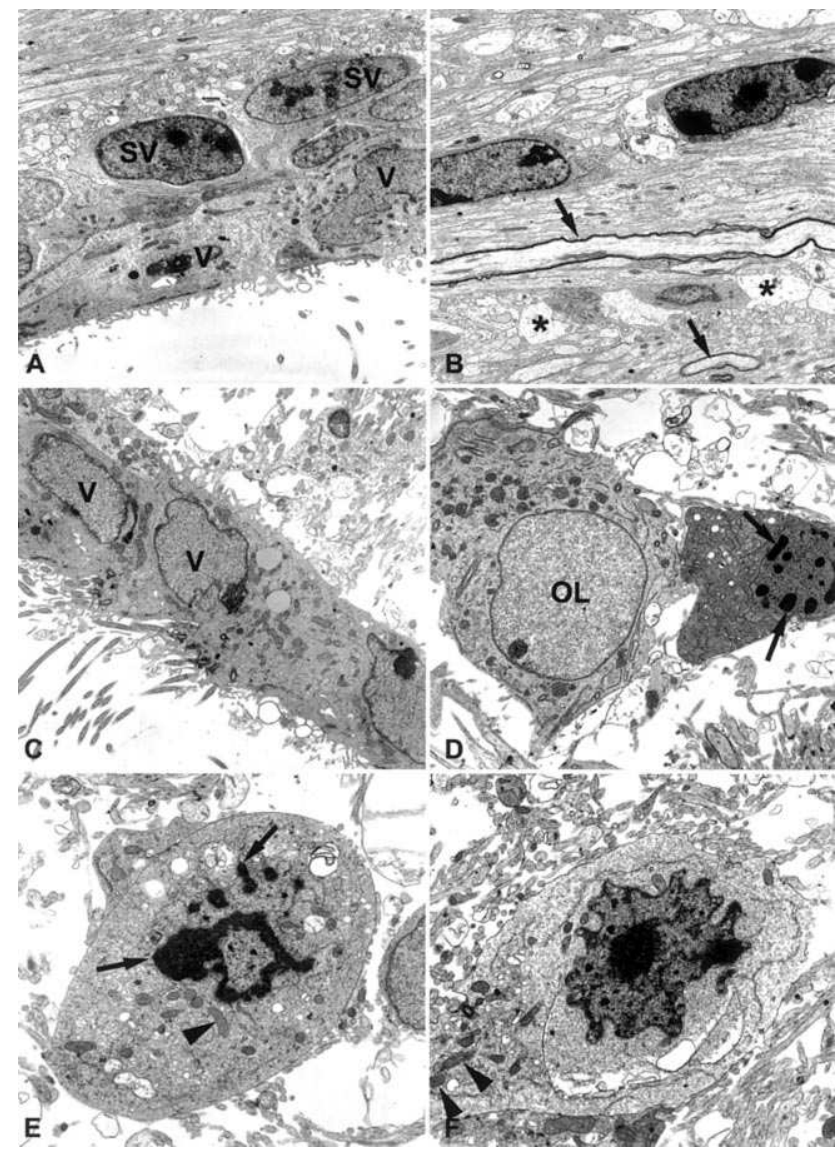

Fig. 4. Ultrathin sections cut from blocks used for preparing Fig. 3B-D. (A-B) Three hours after 70 min of $\mathrm{H}-\mathrm{I}$, the contralateral side appears normal. (A) Ventricular and subventricular cells appeared normal. Subjacent to the subventricular zone, numerous axons run longitudinaly and transversely. Axons were densely packed together, very few degenerating axons were present, and extracellular space was not increased. (B) Deeper in the corpus callosum of the contralateral side, the organization of processes and cells remained normal. The structure of myelin sheaths (arrows) around two axons is typical of axons in the process of being myelinated. The blocks of chromatin are characteristic of immature glia in developing white matter tracts. Astrocytic processes (asterisks) were lightly stained and some contained glycogen particles, an organelle characteristic of immature astrocytes. (C) In the $\mathrm{H}-\mathrm{I}$ hemisphere, ventricular cells formed a continuous layer separating ventricle from brain parenchyma. However, subventricular cells had degenerated. Extracellular space is voluminous and many axons had apparently degenerated. (D) In the injured corpus callosum, a classical apoptotic cell had condensed dense masses of chromatin (arrows), an intact nuclear membrane and mitochondria with intact cristae. An oligodendrocyte (OL) had characteristic features of a myelinating cell found in normal white matter tracts. The cell was identified as a myelinating OL by the presence of many mitochondria, long, numerous cisternae of endoplasmic reticulum, and evenly dispersed chromatin. (E-F) In the injured corpus callosum, two examples of degenerating cells that have features of apoptotic cells. Chromatin (arrows) was condensed into dense masses, nuclear and plasma membranes were mostly intact but showed membrane blebbing around nuclear membrane of one cell (F), and mitochondria had normal cristae (arrowheads). A voluminous extracellular space was filled with many neurites oriented in random directions. $(A, B) \times 4700,(C, F) \times 6600,(D) \times 4400,(E)$ $\times 7400$.

to the cytoplasm and nuclei were unstained, indicating that these cells were viable. These myelin message bearing cells in the subventricular zone were found more

\section{Contralateral}

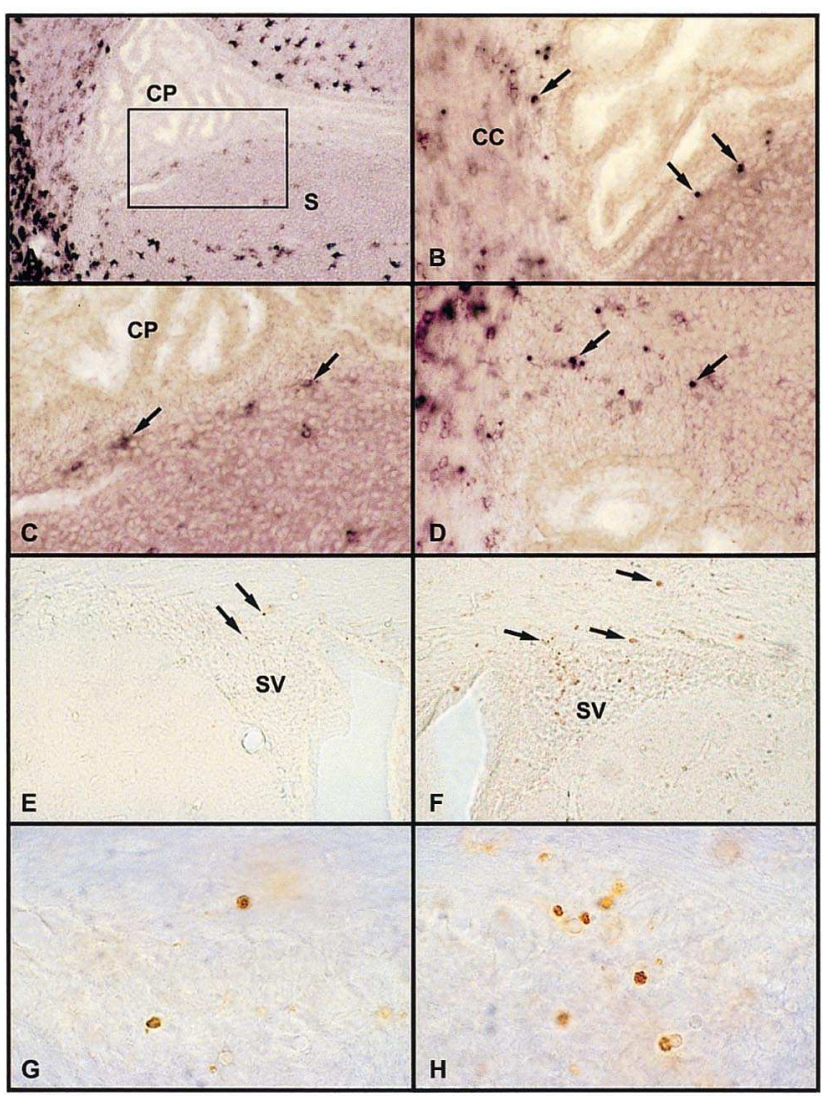

Fig. 5. Vibratomed brain $(50 \mu \mathrm{m})$ sections from $\mathrm{P} 9$ mice processed for PLP in situ hybridization (A-D) and TUNEL (E-H). Tissue samples were obtained $3 \mathrm{~h}$ after $\mathrm{H}-\mathrm{I}$ lesioning. To elicit unilateral forebrain $\mathrm{H}-\mathrm{I}$ injury, animals underwent right carotid artery ligation, followed by 70 min exposure to $10 \%$ oxygen. (A) In the contralateral hemisphere, the intact choroid plexus (CP) was identified in lateral ventricles. Intensely stained $\mathrm{PLP}^{+}$cells were present in striatum (S) and external capsule (left side of picture). (C) Higher magnification of inset showed the subventricular layer with lightly stained $\mathrm{PLP}^{+}$cells (arrows). Message was localized to cytoplasm and nucleus is unstained. (B) Injured side from same animal showed PLP message located in subventricular zone aggregated into small chunks. Nuclei of cells were no longer visible (arrows). (D) Another section from the same side illustrates degenerating OL precursors in subventricular layer (arrows). Many cells in adjacent white matter were intensely stained, some of which appeared to be degenerating. (E) TUNEL stained preparation $3 \mathrm{~h}$ post $\mathrm{H}-\mathrm{I}$ at level of striatum had a few TUNEL $^{+}$cells in subventricular zone in the contralateral hemisphere. $\mathrm{TUNEL}^{+}$cells (arrows) are shown at higher magnification in Fig. 5G. (F) Subventricular zone and corpus callosum in the H-I hemisphere had many $\mathrm{TUNEL}^{+}$cells (arrows). At this time point, very few $\mathrm{TUNEL}^{+}$cells were found in striatum on injured side. $(\mathrm{H})$ Higher magnification shows morphology of $\mathrm{TUNEL}^{+}$cells. Many other TUNEL ${ }^{+}$cells are out of focus due to thickness of section. (A, E, F) $\times 100,(B-D) \times 250,(G, H) \times 500$ 
frequently in the dorsolateral aspect of the ventricle and, more commonly, in the rostral than caudal brain. However, 3 h post $\mathrm{H}-\mathrm{I}$, many degenerating $\mathrm{PLP}^{+}$cells are present in the injured side of the subventricular layer. In the hemisphere ipsilateral to the ligation $3 \mathrm{~h}$ post $\mathrm{H}-\mathrm{I}$, PLP message in the subventricular layer is found in aggregates. Intact $\mathrm{PLP}^{+}$cells were no longer recognizable in the subventricular zone in the injured side; however, most $\mathrm{PLP}^{+}$cells in adjacent white matter tracts retained a normal morphology. These degenerating cells were no longer present in the subventricular zone $24 \mathrm{~h}$ post $\mathrm{H}-\mathrm{I}$, suggesting these OL precursors have degenerated. Coincident with the expression of $\mathrm{PLP}^{+}$degenerating cells in the subventricular zone, increased numbers of TUNEL labeled cells were present in the subventricular zone and corpus callosum (Fig. 5E and F). The subventricular zone in the contralateral hemisphere had several TUNEL labeled cells per section, as expected, but the injured side had several-fold more. Surprisingly, the right striatum may have had a few more TUNEL labeled cells than the contralateral side but the differences were not striking at this time point.

\section{Discussion}

\subsection{Myelin gene expression}

We adapted the well-characterized and widely used unilateral carotid ligation/hypoxia neonatal rat model developed by Vannucci and colleagues (Rice et al., 1981) to elicit forebrain ischemic injury to neonatal mice (Liu et al., 1999). In P9-10 mice, carotid ligation followed by moderate hypoxia $(40-120 \mathrm{~min})$ results, like the rat, in reproducible tissue injury. The severity of injury is determined by the duration of hypoxia exposure. Until recently, studies of neonatal $\mathrm{H}-\mathrm{I}$ have focused primarily upon neuronal, astroglial, and microglial responses to acute ischemic insults and neglected mechanisms of OL injury. This approach is due, in part, to the perception that OL injury is secondary to neuronal injury and, also, in part to the limited availability of appropriate experimental methodology to study OL injury. As far as we are aware, no studies have examined the capacity of OLs to proliferate and myelinate/remyelinate regenerating axons following an $\mathrm{H}-\mathrm{I}$ insult. Our understanding of the role of OLs in $\mathrm{H}-\mathrm{I}$ will be required if we are to understand the complex interactions that transpire between injured neurons and injured myelinating cells. The vulnerabililty of immature OLs to oxygen deprivation and glutamate excitotoxicity have been documented in several recent in vitro studies. These studies point to the possibility that OLs may be direct targets of $\mathrm{H}-\mathrm{I}$ in vivo and, therefore, that these cells may be acutely affected. We compared the pattern of myelin gene expression and glial death in both cerebral hemispheres of 9-day neonatal mice following carotid artery ligation and varying durations of hypoxia. Myelin basic protein and proteolipid protein together constitute $90 \%$ of the protein in myelin, and MBP and PLP transcripts are two of the most abundantly expressed messages in postnatal rodent brain. MBP and PLP gene expression have been successfuly used as sensitive indicators of OL responses in Wallerian degeneration, contusion injury, and other experimental demyelinating conditions (Frei et al., 2000; Jiang et al., 2000). We speculated that changes in expression of these two genes would occur quite rapidly after a significant $\mathrm{H}-\mathrm{I}$ insult. Probing for MBP and PLP transcripts using in situ hybridization, we predictably show that myelin messages are down regulated in ipsilateral white matter tracts $24 \mathrm{~h}$ after right carotid artery ligation and $70 \mathrm{~min}$ of hypoxic exposure. Less expected is the finding that MBP message is visibly decreased in these white matter tracts as early as $3 \mathrm{~h}$ after the same $\mathrm{H}-\mathrm{I}$ regimen. PLP message appears subtly decreased, ipsilateraly, but without additional quantitative studies, we are hesitant to draw the same conclusion for PLP as for MBP. Lack of a pronounced decrease in PLP signal in the ipsilateral side may be due to the fact that the PLP gene encodes for both PLP message and DM20 message, which is an alternative splice product of the PLP gene. Although DM20 is incorporated into the myelin sheath, it is expressed in early embryonic development, and has been postulated by several research groups to have other functions unrelated to myelin sheath compaction (Nadon et al., 1997). These potential functions include transport of molecules to the cell surface and regulation of OL withdrawal from the cell cycle. Therefore, the levels of DM20 transcripts may be regulated post-transcriptionaly in a different way than PLP transcripts. This hypothesis is supported by a recent study showing PLP but not DM20 transcripts decreased after Wallerian degeneration in the peripheral nervous system (Jiang et al., 2000). Preliminary semi-quantitative RT-PCR data of mouse brains $24 \mathrm{~h}$ after an $\mathrm{H}-\mathrm{I}$ insult suggest that PLP but not DM20 transcripts are down regulated in the injured side.

In retrospect, down regulation of myelin gene transcripts $3 \mathrm{~h}$ after lesioning is not surprising in light of our ultrastructural and immunocytochemical studies. The ultrastructural studies provide a level of resolution that is not attainable with other light microscopic techniques. We were surprised at the extensive loss of axons and glia in fornix and corpus callosum in the ipsilateral side that are only noticeable in semi-thin and ultrathin sections. Myelin sheaths are also disintegrating in the ipsilateral side at this early time point as detected with MBP immunocytochemistry. With regard to the MBP immunocytochemistry, the differences between the ipsi- 
lateral and contralateral sides at early time points post $\mathrm{H}-\mathrm{I}$ are not very pronounced at magnifications that show the whole hemisphere. At magnifications above $250 \times$, changes in the structure of the myelin sheath become more noticeable. These findings highlight the sensitivity of the in situ hybridization studies, and show that changes in message levels are a sensitive indicator of changes in OL function. As far as we are aware, this study is the first description documenting changes in myelin gene expression $3 \mathrm{~h}$ after an insult in neonatal rodents. Immediate early gene induction in immature rat brain has been described in both ipsi- and contralateral sides as early as $1 \mathrm{hr}$ following H-I (Ådén et al., 1994; Blumenfeld et al., 1992; Gubits et al., 1993) but as the focus of the studies are in gray matter, it is difficult to know from these studies whether immediate early gene responses occurred in white matter. Evidence for early activation of microglia within 10 min post $\mathrm{H}-\mathrm{I}$ has also been described in cortical gray matter in the corresponding neonatal rat model (Ivacko et al., 1996).

Our studies of neonatal mice show that periventricular white matter is damaged within $3 \mathrm{~h}$ after a moderate $\mathrm{H}-\mathrm{I}$ insult. The corpus callosum and fornix show extensive degenerative changes with loss of axons and neuroglial degeneration. Swift degeneration of axons and glia in the fornix is not surprising as neurons in Ammon's horn show obvious degenerative changes in the semi-thin plastic sections. Degeneration of fibers in the corpus callosum is more surprising as, presumably, most axons in this fiber system arise from cortical neurons. Electron dense cortical neurons, indicative of early degeneration, are obvious but there are not that many at this time point. The contralateral corpus callosum, only 1-2 $\mathrm{mm}$ across the midline, showed little evidence of degeneration at this time point. The apparent lack of degeneration in the contralateral corpus callosum raises the question of whether the $\mathrm{H}-\mathrm{I}$ insult causes direct, acute ipsilateral injury to axons. Secondarily and somewhat later, axons in the contralateral side are likely to degenerate. Another surprising observation is the finding of many neurites in the ipsilateral corpus callosum $3 \mathrm{~h}$ post $\mathrm{H}-\mathrm{I}$. The small caliber of these neurites is diagnostic of growing axons but we do not know whether the neurites represent an early regenerative response from injured neurons or are simply the processes of uninjured neurons that continue to grow through the corpus callosum. In any case, the ultrastructural studies show the white matter is in a dynamic flux containing both degenerating and regenerating fibers. Within $3 \mathrm{~h}$ after $\mathrm{H}-\mathrm{I}$, many different events have already occurred in the corpus callosum and SVZ (see below), and the ultrastructural studies show that early time points, beginning immediately after the cessation of hypoxia, will need to be studied to sort out the sequence of events leading to these degenerative and regenerative changes.
Our studies showing acute degenerative changes in the white matter may be directly relevant for understanding the pathogenesis of human PVL. In humans, neonatal $\mathrm{H}-\mathrm{I}$ white matter injury may have major adverse effect on neuronal development. Compelling recent clinical evidence suggests that secondary neuronal damage occurs relatively rapidly in premature infants following PVL (Inder et al., 1999). Quantitative magnetic resonance imaging was used for longitudinal assessment of cortical and white matter volumes in three groups of infants; premature infants with PVL, premature infants without PVL, and normal term infants. Infants born prematurely, with early white matter injury and who were compared with premature infants without PVL or to normal term infants, had measurable reductions in cortical gray matter volume within 3 months. The volume of total brain myelinated white matter was reduced as well in the infants with PVL (Inder et al., 1999). Together, these findings highlight the complex pathophysiological significance of neonatal white matter injury. One explanation for the subsequent neuronal degeneration observed in PVL is that the $\mathrm{H}-\mathrm{I}$ insult severs the nerves in the region of the ventricles. If this occurs, then both anterograde and retrograde axonal degeneration and subsequent neuronal atrophy may take place over a more prolonged period of time.

\subsection{Subventricular and neuroglial cell death}

Three hours after right carotid artery ligation and 70 min exposure to $10 \% \mathrm{O}_{2}$, we found in the ipsilateral side evidence for cell death in both the subventricular layer and in the adjacent corpus callosum. Cell death was shown by TUNEL staining, in situ hybridization, and ultrastructural studies. While the TUNEL staining and in situ hybridization provide information about the mode of cell death and type of dying cells, examination of the semithin and ultrathin plastic sections add additional information about the swiftness and intensity of the degeneration.

The SVZ, particularly the dorsolateral border, showed degeneration of OL precursors. Intact PLP mRNA $^{+}$cells were absent in the ipsilateral SVZ whereas the contralateral SVZ contained scattered, immature PLP $\mathrm{mRNA}^{+}$cells. In addition, the ipsilateral SVZ dorsal to the ventricle and below the corpus callosum had thinned, suggesting many SVZ cells degenerated before the mice were sacrificed. We have not carefuly examined the medial and lateral borders of the ventricles where the SVZ is missing or thinned out to state whether degeneration has occurred. The cause of cell death in the SVZ including OL precursors located in it could be attributed directly to the $\mathrm{H}-\mathrm{I}$ insult or, possibly, some signal from adjacent axons but this possibility seems less likely because the SVZ cells do not receive neuronal innervation. 
Tissue culture studies support the hypothesis that immature OLs are particularly susceptible to ischemia. Immature OL precursors, identified by the presence of the OL specific 04 antibody, but not mature OLs died within 25 min following oxygen and glucose withdrawal (Fern and Moller, 2000). The mechanism of cell death was mediated by a $\mathrm{Ca}^{2+}$ influx via non-NMDA receptors. We do not know whether $\mathrm{Ca}^{2+}$ fluxes and glutamate excitotoxicity are involved in our in vivo model; however, the $3 \mathrm{~h}$ interval from the end of hypoxic exposure to sacrifice is well within the time frame observed for OL death in culture. The 3-h interval is also adequate for induction of physiological cell death as the number of $\mathrm{TUNEL}^{+}$cells was increased in the ipsi- versus contralateral side. In the ipsilateral corpus callosum and fornix, where most axons have presumably degenerated and regenerating axons are of too small a caliber to be myelinated, we were surprised at the number of OLs that have a large cytoplasm filled with intact mitochondria and endoplasmic reticulum. This phenotype is diagnostic of OLs that are actively myelinating (Skoff et al., 1976a,b). With the exception of some lamellar-like inclusions, most of these OLs appear normal and do not show evidence of degeneration. Our observation that more mature OLs survived in white matter tracts $3 \mathrm{~h}$ after the insult agrees well with the tissue culture studies that show more mature OLs are less susceptible to $\mathrm{O}_{2}$ deprivation. The fate of these more mature OLs in adjacent white matters is unclear. Fewer $\mathrm{MBP}^{+}$and $\mathrm{PLP}^{+}$message bearing cells are present in the corpus callosum and fornix $24 \mathrm{~h}$ post $\mathrm{H}-\mathrm{I}$ than $3 \mathrm{~h}$. The simplest conclusion is that these more mature OLs have degenerated but myelin messages and proteins may be down regulated due to lack of axonal induction. Evidence that myelin messages may be down regulated following an injury and then upregulated following recovery has been shown in a spinal cord injury model. Following spinal cord contusion, the number of $\mathrm{PLP}^{+}$cells declines in and adjacent to the lesion center. By 7 days after injury, the number of $\mathrm{PLP}^{+}$cells was increased adjacent to the lesion, and the authors' comment that the number of $\mathrm{PLP}^{+}$cells increased in the gray matter (Frei et al., 2000). Again, it is not known whether these changes in the gray matter are due to OL proliferation or upregulation of myelin gene expression in pre-existing OLs.

The increase in the number of $\mathrm{TUNEL}^{+}$cells in ipsilateral SVZ and corpus callosum $3 \mathrm{~h}$ post $\mathrm{H}-\mathrm{I}$ along with many degenerating $\mathrm{PLP}^{+}$mRNA cells suggests OLs precursors undergo programmed cell death. This hypothesis is supported by our finding of cells in the corpus callosum with the stereotypic features of apoptotic cells. However, some degenerating cells have properties of both apoptotic and necrotic cells, and we are hesitant to categorize this early glial death as exclusively apoptotic. It is relevant to note that OLs in mice with a mutation in the PLP gene undergo massive cell death and exhibit an apoptotic phenotype in electron microscopic preparations (Knapp et al., 1986). However, the number of TUNEL labeled cells does not begin to match the number of cells with an apoptotic morphology. It is now known that programmed cell death is initiated in certain cells but their DNA is not cleaved into $180 \mathrm{bp}$ fragments in many of these cells (Knapp et al., 1999). Accordingly, TUNEL staining may not be an accurate indicator of the extent of OL death after an $\mathrm{H}-\mathrm{I}$ insult.

Apoptosis of neurons and other neural cell types appears to be more readily elicited in neonatal brain than in adult brain (Han et al., 2000). In a closely related model of focal $\mathrm{HI}$ injury in $\mathrm{P} 7$ rats, treatment with a pan-caspase inhibitor was significantly neuroprotective even when given by intracerebroventricular injection $3 \mathrm{~h}$ post $\mathrm{H}-\mathrm{I}$. In this model, peak pathological caspase activation occured $12-24 \mathrm{~h}$ after lesioning. However, our data indicates that some glial cells are irreversibly injured more rapidly.

There is a distinctive pattern of brain injury that clinicians recognize in the follow-up neuroradiological evaluation of infants who incurred significant $\mathrm{H}-\mathrm{I}$ insults in early infancy; the apparent disappearance of white matter (with compensatory ventricular dilatation) that is unaccompanied by substantial gliosis. We speculate that OL apoptosis results in this pattern of white matter loss, and that the propensity for activation of apoptosis in the injured neonatal brain plays a pivotal role in determining the sequelae of cerebral $\mathrm{H}-\mathrm{I}$.

\subsection{Recovery from an hypoxic-ischemic insult}

The combination of using digoxigenin labeled probes for in situ hybridization and paraformaldehye fixed sections gives excellent cellular resolution and sensitivity. With this technique, we have been able to identify $\mathrm{OL}$ precursors in the $\mathrm{VZ}$ of the brain as early as embryonic day 14. Their detection in the VZ is short lived and by birth, few $\mathrm{PLP}^{+}$OL precursors are located in the VZ. They are now preferentialy congregated in the thickened dorsolateral SVZ. Occasionaly, PLP $\mathrm{mRNA}^{+}$cells are located in the SVZ along the medial and lateral walls of the lateral ventricles. We have not yet observed any in the third ventricle. The intensity of message in these cells is generaly much less than in OLs located in corpus callosum and external capsule. The fate of these cells in normal development is unclear as they may die and/or migrate into surrounding parenchyma. However $3 \mathrm{~h}$ post $\mathrm{H}-\mathrm{I}$ in the ipsilateral side, we found that these $\mathrm{PLP}^{+}$message bearing cells no longer had an intact morphology and their reaction product consisted of aggregates. In our limited examination of $24 \mathrm{~h}$ post $\mathrm{H}-\mathrm{I}$ material, we do not find these $\mathrm{PLP}^{+} \mathrm{OL}$ precursors in both sides of the ventricles. 
This observation suggests that these SVZ cells have degenerated or been signalled to stop producing OL precursors. An unanswered and critical question in this model of $\mathrm{H}-\mathrm{I}$ is whether OL precursors in the SVZ are stimulated to proliferate, migrate, and myelinate surviving axons at later time points. Information regarding the proliferation and myelinating capacity of OLs following an $\mathrm{H}-\mathrm{I}$ insult is minimal and subject to multiple interpretations. An $\mathrm{H}-\mathrm{I}$ study in adult mice suggests that OLs proliferate in the infarcted core (Mandai et al., 1997). Following middle cerebral artery occlusion, the intensity of PLP message in the infarcted core was severely reduced but then increased with time in areas bordering the infarct. Since proliferation assays were not performed, it is unclear whether myelin mRNAs were transiently down-regulated in surviving OLs or whether significant proliferation of OLs and/or their progenitors occurred. A similar response was described following spinal cord injury in the rat wherein MBP message was downregulated for several days but then upregulated near the border of the lesion site (Bartholdi and Schwab, 1998). Within the last decade, the lineages and pluripotentiality of $\mathrm{SV} / \mathrm{SVZ}$ cells has become a focal point of neuroscience research but few studies have looked at whether precursors in the $\mathrm{SV} / \mathrm{SVZ}$ are actively involved in remyelination in an experimentaly altered nervous system. In adult mouse brain, demyelination of the corpus callosum with lysolecithin induced SVZ cells to migrate into the corpus callosum and differentiate into astrocytes and Ols (Nait-Oumesmar et al., 1999). Recent studies show that pluripotential stem cells can be isolated from developing and adult brain, and these cells expanded in tissue culture to form different types of neurons and glia (Barres, 1999; Doetsch et al., 1999; Mehler and Kessler, 1999). Other studies show that the postnatal VZ and SVZ contain astroglial and oligodendroglial precursors that are slowly renewed through a combination of proliferation and death (Levison and Goldman, 1993). The neonatal rodent $\mathrm{H}-\mathrm{I}$ model provides a unique in vivo system to study the plasticity of neuroglial precursors in development. Since the severity of the $\mathrm{H}-\mathrm{I}$ insult in neonates can be experimentaly modified, the plasticity of these $\mathrm{VZ} / \mathrm{SVZ}$ cells can be determined.

\section{Acknowledgements}

The authors wish to acknowledge the technical assistance of Yi-Qing Liu and Haiyan Xu. We thank Dr A.T. and Dr C. Campagnoni for providing the MBP and PLP cDNAs. This research was supported by grants NS35059 (FSS), HD36235 (JDEB), NS38236 (RPS) and NMSS RG2816 (RPS).

\section{References}

Ådén, U., Bona, E., Hagberg, H., Fredholm, B.B., 1994. Changes in c-fos mRNA in the neonatal rat brain following hypoxic ischemia. Neurosci. Lett. 180, 91-95.

Andrews, T., Zhang, P., Bhat, N.R., 1998. TNF alpha potentiates IFN gamma-induced cell death in oligodendrocyte progenitors. J. Neurosci. Res. 54, 574-583.

Back, S.A., Gan, X., Li, Y., Rosenberg, P.A., Volpe, J.J., 1998 Maturation-dependent vulnerability of oligodendrocytes to oxidative stress-induced death caused by glutathione depletion. J. Neurosci. 18, 6241-6253.

Barres, B.A., 1999. A new role for glia: generation of neurons. Cell 97, 667-670.

Bartholdi, D., Schwab, M.E., 1998. Oligodendroglial reaction following spinal cord injury in rat: transient upregulation of MBP mRNA. Glia 23, 278-284.

Bessert, D.A., Skoff, R.P., 1999. High resolution in situ hybridization and TUNEL staining with free-floating brain sections. J. Histochem. Cytochem. 47, 693-701.

Blumenfeld, K.S., Welsh, F.A., Harris, V.A., Pesenson, M.A., 1992. Regional expression of c-fos and Heat Shock Protein-70 mRNA following hypoxia-ischemia in immature rat brain. J. Cerebral. Blood Flow Metab. 12, 987-995.

Dammann, O., Leviton, A., 1997. Maternal intrauterine infection, cytokines, and brain damage in the preterm infant. Pediatr. Res. $42,1-8$.

Doetsch, F., Caille, I., Lim, D.A., Garcia-Verdugo, J.M., AlvarezBuylla, A., 1999. Subventricular zone astrocytes are neural stem cells in the adult mammalian brain. Cell 97, 703-716.

Fern, R., Moller, T., 2000. Rapid ischemic cell death in immature oligodendrocytes: a fatal glutamate release feedback loop. J. Neurosci. 20, 34-42.

Frei, E., Klusman, I., Schnell, L., Schwab, M.E., 2000. Reactions of oligodendrocytes to spinal cord injury: cell survival and myelin repair. Exp. Neurol. 163, 373-380.

Gubits, R.M., Burke, R.E., Casey-McIntosh, G., Bandele, A., Munell, F., 1993. Immediate early gene induction after neonatal hypoxia-ischemia. Mol. Brain Res. 18, 228-238

Han, B.H., D'Costa, A., Back, S.A., Parsadanian, M., Patel, S., Shah, A.R., Gidday, J.M., Srinivasan, A., Deshmukh, M., Holtzman, D.M., 2000. BDNF blocks caspase-3 activation in neonatal hypoxia-ischemia. Neurobiol. Dis. 7, 38-53.

Hartung, H.P., Jung, S., Zielasek, J., Schmidt, B., Archelos, J.J., Toyka, K., 1992. Inflammatory mediators in demyelinating disorders of the CNS and PNS. J. Neuroimmunol. 40, 197-210.

Inder, T.E., Huppi, P.S., Wakefield, S., Kikinis, R., Zientara, G.P., Barnes, P.D., Jolesz, F.A., Volpe, J.J., 1999. Periventricular white matter injury in the premature infant is associated with a reduction in cerebral cortical gray matter volume at term. Ann. Neurol. 46, 755-760.

Ivacko, J.A., Sun, R., Silverstein, F.S., 1996. Hypoxic-ischemic brain injury induces an acute microglial reaction in perinatal rats. Pediatr. Res. 39, 39-47.

Jiang, H., Duchala, C.S., Awatramani, R., Shumas, S., Carlock, L., Kamholz, J., Garbern, J., Scherer, S.S., Shy, M.E., Macklin, W.B., 2000. Proteolipid protein mRNA stability is regulated by axonal contact in the rodent peripheral nervous system. J. Neurobiol. 44, 7-19.

Knapp, P., Bartlett, W., Williams, L., Yamada, M., Ikenaka, K., Skoff, R., 1999. Programmed cell death without DNA fragmentation in the jimpy mouse: secreted factors can enhance survival. Cell Death Diff. 6, 136-145.

Knapp, P.E., Skoff, R.P., Redstone, D.W., 1986. Oligodendroglial cell death in jimpy mice: an explanation for the myelin deficit. J. Neurosci. 6, 2813-2822. 
Levison, S.W., Goldman, J.E., 1993. Both oligodendrocytes and astrocytes develop from progenitors in the subventricular zone of postnatal rat forebrain. Neuron 10, 201-212.

Liu, X.H., Kwon, D., Schielke, G., Yang, G.Y., Silverstein, F.S., Barks, J.D.E., 1999. Mice deficient in IL-1 converting enzyme are resistant to neonatal hypoxic-ischemic brain damage. J. Cereb. Blood Flow Metab. 19, 1099-1108.

Mandai, K., Matsumoto, M., Kitagawa, K., Matsushita, K., Ohtsuki, T., Mabuchi, T., Colman, D.R., 1997. Ischemic damage and subsequent proliferation of oligodendrocytes in focal cerebral ischemia. Neuroscience 77, 849-861.

Mehler, M.F., Kessler, J.A., 1999. Progenitor cell biology: implications for neural regeneration. Arch. Neurol. 56, 80-784.

Nadon, N.L., Miller, S., Draeger, K., Salvggio, M., 1997. Myelin proteolipid DM20: evidence for function independent of myelination. J. Dev. Neurosci. 15, 285-293.

Nait-Oumesmar, B., Decker, L., Lachapelle, F, Avellana-Adalid, V., Bachelin, C., Baron-Van Evercooren, A., 1999. Progenitor cells of the adult mouse subventricular zone proliferate, migrate and differentiate into oligodendrocytes after demyelination. Eur. J. Neurosci. 11, 4357-4366.
Petito, C.K., Olarte, J.P., Roberts, B., Nowak, T.S., Jr, Pulsinelli, W.A., 1998. Selective glial vulnerability following transient global ischemia in rat brain. J. Neuropathol. Exp. Neurol. 57, 231-238.

Rice, J.E., Vannucci, R.C., Brierley, J.B., 1981. The influence of immaturity on hypoxic-ischemic brain damage in the rat. Ann. Neurol. 9, 131-141.

Silverstein F.S., Song D., Xu H., Barks J., G. Schielke G., Skoff R., 2000. Resistance to hypoxic-ischemic oligodendroglial injury in neonatal interleukin-1 converting enzyme deficient mice, Soc. Neurosci. Abstr., 26(2), 2065

Skoff, R.P., Price, D.L., Stocks, A., 1976a. Electron microscopic autoradiographic studies of gliogenesis in rat optic nerve. I. Cell proliferation. J. Comp. Neurol 169, 291-312.

Skoff, R.P., Price, D.L., Stocks, A., 1976b. Electron microscopic autoradiographic studies of gliogenesis in rat optic nerve. II. Time of origin. J. Comp. Neurol 169, 313-334.

Xu, H., Liu, Y.C., Skoff, R., Barks, J.D., Silverstein, F.S., 2000. Acute oligodendroglial injury in neonatal rodent brain. Pediatr. Res. 47, 467A.

Volpe, J.J., 1999. Neurologic outcome of prematurity. Arch. Neurol. 55, 297-300. 